\title{
Fusion for AdS/CFT boundary S-matrices
}

\author{
Rafael I. Nepomechie ${ }^{a}$ and Rodrigo A. Pimenta ${ }^{a, b}$ \\ ${ }^{a}$ Physics Department, University of Miami, \\ P.O. Box 248046, Coral Gables, FL 33124 U.S.A. \\ ${ }^{b}$ Departamento de Fúsica, Universidade Federal de São Carlos, \\ Caixa Postal 676, CEP 13569-905, São Carlos, Brasil \\ E-mail: nepomechie@physics.miami.edu, pimenta@df.ufscar.br
}

ABSTRACT: We propose a fusion formula for AdS/CFT worldsheet boundary S-matrices. We show that, starting from the fundamental $Y=0$ boundary S-matrix, this formula correctly reproduces the two-particle bound-state boundary S-matrices.

Keywords: AdS-CFT Correspondence, Boundary Quantum Field Theory, Exact SMatrix, Integrable Field Theories

ARXIV EPRINT: 1509.02426 


\section{Contents}

1 Introduction 1

2 Bulk fusion $\quad 2$

2.1 AdS/CFT bulk S-matrix: symmetric representation 3

2.2 AdS/CFT bulk S-matrix: antisymmetric representation 6

3 Boundary fusion $\quad 7$

3.1 AdS/CFT boundary S-matrix: symmetric representation 8

3.2 AdS/CFT boundary S-matrix: antisymmetric representation 9

4 Discussion $\quad 9$

$\begin{array}{ll}\text { A Fundamental bulk S-matrix } & 10\end{array}$

$\begin{array}{ll}\text { B Proof of the boundary fusion formulas } & 11\end{array}$

\section{Introduction}

The formation of bound states ("fusion") is a ubiquitous phenomenon in quantum field theory. If the theory is integrable [1], then the factorized bulk S-matrices of the bound-state particles can be determined in terms of the corresponding S-matrices of the fundamental particles [2]. This phenomenon was abstracted in [3, 4] into a general "fusion procedure" for constructing higher-dimensional R-matrices (solutions of the Yang-Baxter equation) starting from a fundamental R-matrix. For boundary S-matrices/K-matrices, i.e. solutions of the boundary Yang-Baxter equation [5-7], an analogous fusion procedure was formulated in $[8-10]$.

In order to carry out the fusion procedure [3], the fundamental R-matrix should satisfy a certain technical requirement: namely, it must degenerate into a projection operator for some value(s) of the spectral parameter. Many R-matrices fulfill this requirement; and this fusion procedure has proved to be very useful: it not only generates new solutions of the Yang-Baxter equation, but it also leads to a hierarchy of commuting transfer matrices that can be used to solve the corresponding integrable models (see e.g. [11]).

However, the AdS/CFT worldsheet bulk S-matrix [12, 13], which plays a key role in the understanding of integrability in AdS/CFT [14], does not satisfy this requirement. This apparent failure of the fusion procedure has been quite puzzling, since bound states do form in this model $[15,16]$, and their bulk S-matrices have been determined [17, 18], albeit by other means.

This puzzle was recently resolved by Beisert, de Leeuw and Nag [19], who showed that one can relax the requirement that the R-matrix degenerates into a projector. In particular, 
they proposed a new bulk fusion formula, which generates a bound-state AdS/CFT bulk S-matrix [17] from the fundamental one. As a bonus, the resulting fused matrix automatically has the correct dimensions - the additional similarity transformation and subsequent elimination of null rows and columns that are implicit in the original approach [3] are not needed. (A similar fusion formula was proposed for the XXX R-matrix in [20].)

Factorized boundary S-matrices also play an interesting role in AdS/CFT (see e.g. [21, $22]$ ); and AdS/CFT boundary S-matrices for bound states have also been determined [2325]. (See also [26-31] and references therein.) The main purpose of this note is to propose a new fusion formula for boundary S-matrices, which generates two-particle bound-state AdS/CFT boundary S-matrices from the fundamental one.

The outline of this paper is as follows. In section 2 we briefly review the new bulk fusion procedure formulated in [19]. However, we work with different conventions, which we find more convenient. In section 3 we present the corresponding boundary fusion formula, whose proof is relegated to an appendix. We then show that, starting from the fundamental $Y=0$ boundary S-matrix [21], this formula correctly reproduces the bound-state boundary S-matrices found in [24] and [23], respectively. We conclude in section 4 with a brief discussion of our results.

\section{Bulk fusion}

We begin by briefly reviewing the new fusion procedure proposed in [19]. We consider an R-matrix $R\left(z_{1}, z_{2}\right)$

$$
R\left(z_{1}, z_{2}\right): \quad \mathcal{C}^{n} \otimes \mathcal{C}^{n} \mapsto \mathcal{C}^{n} \otimes \mathcal{C}^{n},
$$

which is a solution of the (graded) Yang-Baxter equation

$$
R_{12}\left(z_{1}, z_{2}\right) R_{13}\left(z_{1}, z_{3}\right) R_{23}\left(z_{2}, z_{3}\right)=R_{23}\left(z_{2}, z_{3}\right) R_{13}\left(z_{1}, z_{3}\right) R_{12}\left(z_{1}, z_{2}\right),
$$

where $R_{12}\left(z_{1}, z_{2}\right)=R\left(z_{1}, z_{2}\right) \otimes \mathbb{I}_{n}, R_{13}\left(z_{1}, z_{3}\right)=\mathcal{P}_{23} R_{12}\left(z_{1}, z_{3}\right) \mathcal{P}_{23}, R_{23}\left(z_{2}, z_{3}\right)=$ $\mathcal{P}_{12} R_{13}\left(z_{2}, z_{3}\right) \mathcal{P}_{12}$, and $\mathcal{P}$ denotes the (graded) permutation matrix

$$
\mathcal{P}=\sum_{a, b=1}^{n}(-1)^{\epsilon_{a} \epsilon_{b}} e_{a b} \otimes e_{b a}, \quad\left(e_{a b}\right)_{i j}=\delta_{a, i} \delta_{b, j},
$$

where $\epsilon_{a} \in\{0,1\}$ are the gradings.

We further suppose that a bound state forms for certain rapidities $\left(z_{1}, z_{2}\right)$; and correspondingly, $R\left(z_{1}, z_{2}\right)$ drops in rank to $m<n^{2}$, and admits the following important decomposition [19]

$$
R\left(z_{1}, z_{2}\right)=\mathcal{E}\left(z_{1}, z_{2}\right) \mathcal{H}\left(z_{1}, z_{2}\right) \mathcal{F}\left(z_{1}, z_{2}\right), \quad \mathcal{F}\left(z_{1}, z_{2}\right) \mathcal{E}\left(z_{1}, z_{2}\right)=\mathbb{I}_{m}
$$

where the matrices act as follows

$$
\begin{aligned}
\mathcal{E}\left(z_{1}, z_{2}\right): & & \mathcal{C}^{m} \mapsto \mathcal{C}^{n} \otimes \mathcal{C}^{n}, \\
\mathcal{H}\left(z_{1}, z_{2}\right): & & \mathcal{C}^{m} \mapsto \mathcal{C}^{m},
\end{aligned}
$$




$$
\mathcal{F}\left(z_{1}, z_{2}\right): \mathcal{C}^{n} \otimes \mathcal{C}^{n} \mapsto \mathcal{C}^{m}
$$

Note that $\mathcal{E} \mathcal{F}$ is a projector

$$
\left[\mathcal{E}\left(z_{1}, z_{2}\right) \mathcal{F}\left(z_{1}, z_{2}\right)\right]^{2}=\mathcal{E}\left(z_{1}, z_{2}\right) \mathcal{F}\left(z_{1}, z_{2}\right)
$$

hence (2.3) means that $R\left(z_{1}, z_{2}\right)$ is "almost" (i.e., up to the factor $\left.\mathcal{H}\left(z_{1}, z_{2}\right)\right)$ a projector. It it evident from the decomposition (2.3) that [19]

$$
\begin{aligned}
R\left(z_{1}, z_{2}\right) \mathcal{E}\left(z_{1}, z_{2}\right) & =\mathcal{E}\left(z_{1}, z_{2}\right) \mathcal{H}\left(z_{1}, z_{2}\right), \\
\mathcal{F}\left(z_{1}, z_{2}\right) R\left(z_{1}, z_{2}\right) & =\mathcal{H}\left(z_{1}, z_{2}\right) \mathcal{F}\left(z_{1}, z_{2}\right), \\
R\left(z_{1}, z_{2}\right) \mathcal{E}\left(z_{1}, z_{2}\right) \mathcal{F}\left(z_{1}, z_{2}\right) & =R\left(z_{1}, z_{2}\right) .
\end{aligned}
$$

These "fusion identities" can be used to show that the fused R-matrices [19]

$$
\begin{aligned}
& R_{\langle 12\rangle 3}\left(z_{1}, z_{2} \mid z_{3}\right)=\mathcal{F}_{\langle 12\rangle}\left(z_{1}, z_{2}\right) R_{13}\left(z_{1}, z_{3}\right) R_{23}\left(z_{2}, z_{3}\right) \mathcal{E}_{\langle 12\rangle}\left(z_{1}, z_{2}\right), \\
& R_{1\langle 23\rangle}\left(z_{1} \mid z_{2}, z_{3}\right)=\mathcal{F}_{\langle 23\rangle}\left(z_{2}, z_{3}\right) R_{13}\left(z_{1}, z_{3}\right) R_{12}\left(z_{1}, z_{2}\right) \mathcal{E}_{\langle 23\rangle}\left(z_{2}, z_{3}\right),
\end{aligned}
$$

obey corresponding fused (graded) Yang-Baxter equations. Although these R-matrices are generally not symmetric, they can be made so by a similarity transformation [19]

$$
\begin{aligned}
& R_{\langle 12\rangle 3}^{\prime}\left(z_{1}, z_{2} \mid z_{3}\right)=W_{\langle 12\rangle}\left(z_{1}, z_{2}\right) R_{\langle 12\rangle 3}\left(z_{1}, z_{2} \mid z_{3}\right) W_{\langle 12\rangle}^{-1}\left(z_{1}, z_{2}\right), \\
& R_{1\langle 23\rangle}^{\prime}\left(z_{1} \mid z_{2}, z_{3}\right)=W_{\langle 23\rangle}\left(z_{2}, z_{3}\right) R_{1\langle 23\rangle}\left(z_{1} \mid z_{2}, z_{3}\right) W_{\langle 23\rangle}^{-1}\left(z_{2}, z_{3}\right),
\end{aligned}
$$

where $W^{T}\left(z_{1}, z_{2}\right) W\left(z_{1}, z_{2}\right)=\mathcal{H}\left(z_{1}, z_{2}\right)$.

Complementary operators $\overline{\mathcal{E}}$ and $\overline{\mathcal{F}}$ satisfying [19]

$$
\mathcal{F}\left(z_{1}, z_{2}\right) \overline{\mathcal{E}}\left(z_{1}, z_{2}\right)=0, \quad \overline{\mathcal{F}}\left(z_{1}, z_{2}\right) \mathcal{E}\left(z_{1}, z_{2}\right)=0, \quad \overline{\mathcal{F}}\left(z_{1}, z_{2}\right) \overline{\mathcal{E}}\left(z_{1}, z_{2}\right)=\mathbb{I}_{n^{2}-m}
$$

as well as the completeness relation

$$
\mathcal{E}\left(z_{1}, z_{2}\right) \mathcal{F}\left(z_{1}, z_{2}\right)+\overline{\mathcal{E}}\left(z_{1}, z_{2}\right) \overline{\mathcal{F}}\left(z_{1}, z_{2}\right)=\mathbb{I}_{n^{2}}
$$

can be used to construct corresponding complementary fused R-matrices

$$
\begin{aligned}
& \bar{R}_{\langle\overline{12\rangle}\rangle 3}\left(z_{1}, z_{2} \mid z_{3}\right)=\overline{\mathcal{F}}_{\langle\overline{12\rangle}}\left(z_{1}, z_{2}\right) R_{13}\left(z_{1}, z_{3}\right) R_{23}\left(z_{2}, z_{3}\right) \overline{\mathcal{E}}_{\langle\overline{12\rangle}\rangle}\left(z_{1}, z_{2}\right), \\
& \bar{R}_{1\langle\overline{23\rangle}\rangle}\left(z_{1} \mid z_{2}, z_{3}\right)=\overline{\mathcal{F}}_{\langle\overline{23\rangle}\rangle}\left(z_{2}, z_{3}\right) R_{13}\left(z_{1}, z_{3}\right) R_{12}\left(z_{1}, z_{2}\right) \overline{\mathcal{E}}_{\langle\overline{23\rangle}}\left(z_{2}, z_{3}\right) .
\end{aligned}
$$

\subsection{AdS/CFT bulk S-matrix: symmetric representation}

Let us now apply this formalism to one copy of the fundamental su(2|2) AdS/CFT bulk S-matrix. To this end, we set

$$
R\left(z_{1}, z_{2}\right)=S^{A A}\left(z_{1}, z_{2}\right)
$$

as given by Arutyunov and Frolov in [17], which is reproduced in appendix A for the reader's convenience. This S-matrix satisfies the graded Yang-Baxter equation (2.1) with the gradings $\epsilon_{1}=\epsilon_{2}=0, \epsilon_{3}=\epsilon_{4}=1$. 
We use an elliptic parametrization for the momentum $p$ and the parameters $x^{ \pm}$for $M$-particle bound states [16, 17]

$$
p(z)=2 \operatorname{am}(z, k), \quad x^{ \pm}(z)=\frac{M}{2 g}\left(\frac{\operatorname{cn}(z, k)}{\operatorname{sn}(z, k)} \pm i\right)(1+\operatorname{dn}(z, k)), \quad k=-\frac{4 g^{2}}{M^{2}},
$$

such that

$$
\frac{x^{+}}{x^{-}}=e^{i p}
$$

and

$$
x^{+}+\frac{1}{x^{+}}-x^{-}-\frac{1}{x^{-}}=\frac{2 M i}{g},
$$

where $g>0$ is the coupling constant. However, we henceforth reserve $p$ and $x^{ \pm}$for the momentum and parameters of the fundamental particles $(M=1)$, and $P$ and $y^{ \pm}$for the corresponding quantities of the two-particle bound states $(M=2)$.

Consider a pair of fundamental particles with parameters $x_{i}^{ \pm}=x^{ \pm}\left(z_{i}\right), i=1,2$. These particles form a bound state when $[15,16]^{1}$

$$
x_{1}^{-}=x_{2}^{+} .
$$

Indeed, adding the two constraint equations (2.19)

$$
\begin{aligned}
& x_{1}^{+}+\frac{1}{x_{1}^{+}}-x_{1}^{-}-\frac{1}{x_{1}^{-}}=\frac{2 i}{g}, \\
& x_{2}^{+}+\frac{1}{x_{2}^{+}}-x_{2}^{-}-\frac{1}{x_{2}^{-}}=\frac{2 i}{g},
\end{aligned}
$$

imposing the fusion condition (2.20), and making the identifications

$$
y^{+}=x_{1}^{+}, \quad y^{-}=x_{2}^{-},
$$

we arrive at the two-particle bound-state constraint

$$
y^{+}+\frac{1}{y^{+}}-y^{-}-\frac{1}{y^{-}}=\frac{4 i}{g} .
$$

Note that the momentum of the bound state is indeed the sum of the momenta of its constituents, since

$$
e^{i P}=\frac{y^{+}}{y^{-}}=\frac{x_{1}^{+}}{x_{2}^{-}}=\frac{x_{1}^{+}}{x_{1}^{-}} \frac{x_{2}^{+}}{x_{2}^{-}}=e^{i\left(p_{1}+p_{2}\right)},
$$

where $p_{i}=p\left(z_{i}\right)$. This bound state lies in the 8-dimensional symmetric representation of $s u(2 \mid 2)[32]$.

When the fusion condition (2.20) is satisfied, the rank of $R\left(z_{1}, z_{2}\right)$ drops from 16 to 8 . By determining the normalized eigenvectors corresponding to the nonzero eigenvalues, we obtain the decomposition (2.3) with

$$
\mathcal{E}\left(z_{1}, z_{2}\right)=\tilde{\mathcal{E}}\left(z_{1}, z_{2}\right) N^{-1}\left(z_{1}, z_{2}\right),
$$

\footnotetext{
${ }^{1}$ We also assume [16] that $\left|x_{i}^{ \pm}\right|>1$ and $\left(x_{1}^{+}\right)^{*}=x_{2}^{-}$.
} 
where

$$
\tilde{\mathcal{E}}\left(z_{1}, z_{2}\right)=\left(\begin{array}{cccccccc}
0 & 0 & 1 & 0 & 0 & 0 & 0 & 0 \\
0 & \frac{1}{\sqrt{2}} & 0 & -\frac{1}{\sqrt{2}} a_{2} & 0 & 0 & 0 & 0 \\
0 & 0 & 0 & 0 & 0 & 0 & 0 & a_{5} \\
0 & 0 & 0 & 0 & 0 & a_{5} & 0 & 0 \\
0 & \frac{1}{\sqrt{2}} & 0 & \frac{1}{\sqrt{2}} a_{2} & 0 & 0 & 0 & 0 \\
1 & 0 & 0 & 0 & 0 & 0 & 0 & 0 \\
0 & 0 & 0 & 0 & 0 & 0 & a_{5} & 0 \\
0 & 0 & 0 & 0 & a_{5} & 0 & 0 & 0 \\
0 & 0 & 0 & 0 & 0 & 0 & 0 & a_{10} \\
0 & 0 & 0 & 0 & 0 & 0 & a_{10} & 0 \\
0 & 0 & 0 & 0 & 0 & 0 & 0 & 0 \\
0 & 0 & 0 & -\sqrt{2} a_{8} & 0 & 0 & 0 & 0 \\
0 & 0 & 0 & 0 & 0 & a_{10} & 0 & 0 \\
0 & 0 & 0 & 0 & a_{10} & 0 & 0 & 0 \\
0 & 0 & 0 & \sqrt{2} a_{8} & 0 & 0 & 0 & 0 \\
0 & 0 & 0 & 0 & 0 & 0 & 0 & 0
\end{array}\right),
$$

and $N\left(z_{1}, z_{2}\right)$ is the diagonal matrix

$$
N\left(z_{1}, z_{2}\right)=\operatorname{diag}\left(1,1,1, n_{1}, n_{2}, n_{2}, n_{2}, n_{2}\right), \quad n_{1}=\sqrt{a_{2}^{2}+4 a_{8}^{2}}, \quad n_{2}=\sqrt{a_{5}^{2}+a_{10}^{2}},
$$

where the $a_{k}=a_{k}\left(z_{1}, z_{2}\right)$ are given by (A.3). Moreover,

$$
\mathcal{F}\left(z_{1}, z_{2}\right)=\mathcal{E}^{T}\left(z_{1}, z_{2}\right)=N^{-1}\left(z_{1}, z_{2}\right) \tilde{\mathcal{E}}^{T}\left(z_{1}, z_{2}\right)=N^{-1}\left(z_{1}, z_{2}\right) \tilde{\mathcal{F}}\left(z_{1}, z_{2}\right)
$$

where we have defined $\tilde{\mathcal{F}}\left(z_{1}, z_{2}\right)=\tilde{\mathcal{E}}^{T}\left(z_{1}, z_{2}\right)$. Finally,

$$
\mathcal{H}\left(z_{1}, z_{2}\right)=\operatorname{diag}\left(1,1,1, h_{1}, h_{2}, h_{2}, h_{2}, h_{2}\right), \quad h_{1}=a_{2}+a_{4}, \quad h_{2}=a_{5}+a_{6} .
$$

Performing the similarity transformation (2.11) with the matrix

$$
W\left(z_{1}, z_{2}\right)=\left(\begin{array}{cccccccc}
0 & 0 & 1 & 0 & 0 & 0 & 0 & 0 \\
0 & 1 & 0 & 0 & 0 & 0 & 0 & 0 \\
1 & 0 & 0 & 0 & 0 & 0 & 0 & 0 \\
0 & 0 & 0 & w_{1} & 0 & 0 & 0 & 0 \\
0 & 0 & 0 & 0 & 0 & 0 & 0 & w_{2} \\
0 & 0 & 0 & 0 & 0 & w_{2} & 0 & 0 \\
0 & 0 & 0 & 0 & 0 & 0 & w_{2} & 0 \\
0 & 0 & 0 & 0 & w_{2} & 0 & 0 & 0
\end{array}\right), \quad w_{1}=\sqrt{a_{2}+a_{4}}, \quad w_{2}=\sqrt{a_{5}+a_{6}},
$$

we obtain

$$
R_{1\langle 23\rangle}^{\prime}\left(z_{1} \mid z_{2}, z_{3}\right)=U_{\langle 23\rangle}\left(z_{2}, z_{3}\right) \tilde{\mathcal{F}}_{\langle 23\rangle}\left(z_{2}, z_{3}\right) R_{13}\left(z_{1}, z_{3}\right) R_{12}\left(z_{1}, z_{2}\right) \tilde{\mathcal{E}}_{\langle 23\rangle}\left(z_{2}, z_{3}\right) V_{\langle 23\rangle}^{-1}\left(z_{2}, z_{3}\right)
$$

where we have defined the new matrices $U$ and $V$, which evidently have the same matrix structure as $W$, but have different matrix elements

$$
U\left(z_{1}, z_{2}\right)=W\left(z_{1}, z_{2}\right) N^{-1}\left(z_{1}, z_{2}\right)=\left.W\left(z_{1}, z_{2}\right)\right|_{w_{i} \rightarrow u_{i}},
$$




$$
V\left(z_{1}, z_{2}\right)=W\left(z_{1}, z_{2}\right) N\left(z_{1}, z_{2}\right)=\left.W\left(z_{1}, z_{2}\right)\right|_{w_{i} \rightarrow v_{i}}
$$

By explicit computation we obtain the following results for these matrix elements

$$
\begin{aligned}
& u_{1}=\frac{w_{1}}{n_{1}}=\left[2 i g \sin \left(p_{1} / 2\right) \sin \left(p_{2} / 2\right)\right]^{-1} \\
& u_{2}=\frac{w_{2}}{n_{2}}=e^{-i p_{1} / 2} \frac{\eta\left(z_{12}, 2\right)}{\eta\left(z_{2}, 1\right)}, \\
& v_{1}=w_{1} n_{1}=8 i g \frac{\sin ^{2}\left(p_{1} / 2\right) \sin ^{2}\left(p_{2} / 2\right)}{\sin ^{2}\left(\left(p_{1}+p_{2}\right) / 2\right)}\left(1-g^{2} \sin \left(p_{1} / 2\right) \sin \left(p_{2} / 2\right) \sin ^{2}\left(\left(p_{1}+p_{2}\right) / 2\right),\right. \\
& v_{2}=w_{2} n_{2}=\left[\left(1+e^{-i\left(p_{1}+p_{2}\right) / 2}\right)^{-1}+e^{i p_{1}}\left(1+e^{i\left(p_{1}+p_{2}\right) / 2}\right)^{-1}\right] \frac{\eta\left(z_{2}, 1\right)}{\eta\left(z_{12}, 2\right)},
\end{aligned}
$$

where $\eta(z, M)$ is defined in (A.5), and the rapidity $z_{12}$ is defined such that

$$
y^{+}\left(z_{12}\right)=x^{+}\left(z_{1}\right), \quad y^{-}\left(z_{12}\right)=x^{-}\left(z_{2}\right),
$$

as in (2.22). Remarkably, the square roots in $u_{i}$ and $v_{i}$ (recall the definitions of $n_{i}$ and $w_{i}$ given in (2.27) and (2.30)) have all disappeared.

Using these results to evaluate (2.31), we have verified numerically that this fused R-matrix coincides with $S^{A B}$ in $[17]^{2}$

$$
S^{A B}\left(z_{1}, z_{23}\right)=R_{1\langle 23\rangle}^{\prime}\left(z_{1} \mid z_{2}, z_{3}\right) .
$$

A similar result was argued in [19].

\subsection{AdS/CFT bulk S-matrix: antisymmetric representation}

We now proceed to construct the complementary fused S-matrix (2.15), which corresponds to the antisymmetric representation of $s u(2 \mid 2)$ [32], which is also 8-dimensional. The required complementary operators $\overline{\mathcal{E}}$ and $\overline{\mathcal{F}}$ can be obtained by considering the "opposite" fusion condition

$$
x_{1}^{+}=x_{2}^{-} .
$$

Since all the $a_{k}$ (A.3) except $a_{1}$ have a simple pole at this point, it is convenient to introduce rescaled quantities $\hat{a}_{k}\left(z_{1}, z_{2}\right)=\left(x_{1}^{+}-x_{2}^{-}\right) a_{k}\left(z_{1}, z_{2}\right)$ and $\hat{R}\left(z_{1}, z_{2}\right)=\left(x_{1}^{+}-x_{2}^{-}\right) S^{A A}\left(z_{1}, z_{2}\right)$.

When the fusion condition (2.36) is satisfied, the rank of $\hat{R}\left(z_{1}, z_{2}\right)$ indeed drops from 16 to 8 , and we obtain the decomposition

$$
\hat{R}\left(z_{1}, z_{2}\right)=\mathcal{E}_{\mathcal{A}}\left(z_{1}, z_{2}\right) \mathcal{H}_{\mathcal{A}}\left(z_{1}, z_{2}\right) \mathcal{F}_{\mathcal{A}}\left(z_{1}, z_{2}\right), \quad \mathcal{F}_{\mathcal{A}}\left(z_{1}, z_{2}\right) \mathcal{E}_{\mathcal{A}}\left(z_{1}, z_{2}\right)=\mathbb{I}_{8},
$$

with

$$
\mathcal{E}_{\mathcal{A}}\left(z_{1}, z_{2}\right)=\tilde{\mathcal{E}}_{\mathcal{A}}\left(z_{1}, z_{2}\right) N^{-1}\left(z_{1}, z_{2}\right)
$$

\footnotetext{
${ }^{2}$ As noted in [24], there are two typos in the coefficients of $S^{A B}$ listed in section 6.1.2 of [17]. In $a_{13}$, the factor in the numerator $\left(x_{1}^{-}-y_{2}^{+}\right)$should be instead $\left(x_{1}^{+}-y_{2}^{+}\right)$; i.e., the $x_{1}^{-}$should be changed to $x_{1}^{+}$. And in $a_{14}$, the factor in the numerator $\left(1-y_{2}^{-} x_{1}^{-}\right)$should be instead $\left(1-y_{2}^{-} x_{1}^{+}\right)$; i.e., the $x_{1}^{-}$should be changed to $x_{1}^{+}$.
} 
where

$$
\tilde{\mathcal{E}}_{\mathcal{A}}\left(z_{1}, z_{2}\right)=\left(\begin{array}{cccccccc}
0 & 0 & 0 & 0 & 0 & 0 & 0 & 0 \\
0 & 0 & 0 & -\frac{1}{\sqrt{2}} a_{2} & 0 & 0 & 0 & 0 \\
0 & 0 & 0 & 0 & 0 & 0 & 0 & a_{5} \\
0 & 0 & 0 & 0 & 0 & a_{5} & 0 & 0 \\
0 & 0 & 0 & \frac{1}{\sqrt{2}} a_{2} & 0 & 0 & 0 & 0 \\
0 & 0 & 0 & 0 & 0 & 0 & 0 & 0 \\
0 & 0 & 0 & 0 & 0 & 0 & a_{5} & 0 \\
0 & 0 & 0 & 0 & a_{5} & 0 & 0 & 0 \\
0 & 0 & 0 & 0 & 0 & 0 & 0 & a_{10} \\
0 & 0 & 0 & 0 & 0 & 0 & a_{10} & 0 \\
0 & 0 & 1 & 0 & 0 & 0 & 0 & 0 \\
0 & \frac{1}{\sqrt{2}} & 0 & -\sqrt{2} a_{8} & 0 & 0 & 0 & 0 \\
0 & 0 & 0 & 0 & 0 & a_{10} & 0 & 0 \\
0 & 0 & 0 & 0 & a_{10} & 0 & 0 & 0 \\
0 & \frac{1}{\sqrt{2}} & 0 & \sqrt{2} a_{8} & 0 & 0 & 0 & 0 \\
1 & 0 & 0 & 0 & 0 & 0 & 0 & 0
\end{array}\right),
$$

and $N\left(z_{1}, z_{2}\right)$ is again given by (2.27). (Note that the singular factors in $N\left(z_{1}, z_{2}\right)$ and $\tilde{\mathcal{E}}_{\mathcal{A}}\left(z_{1}, z_{2}\right)$ are canceled in $\mathcal{E}_{\mathcal{A}}\left(z_{1}, z_{2}\right)$.) Moreover, $\mathcal{F}_{\mathcal{A}}\left(z_{1}, z_{2}\right)=\mathcal{E}_{\mathcal{A}}^{T}\left(z_{1}, z_{2}\right)$, and

$$
\mathcal{H}_{\mathcal{A}}\left(z_{1}, z_{2}\right)=\operatorname{diag}\left(\hat{a}_{3}, \hat{a}_{3}, \hat{a}_{3}, \hat{h}_{1}, \hat{h}_{2}, \hat{h}_{2}, \hat{h}_{2}, \hat{h}_{2}\right), \quad \hat{h}_{1}=\hat{a}_{2}+\hat{a}_{4}, \quad \hat{h}_{2}=\hat{a}_{5}+\hat{a}_{6} .
$$

Finally, the complementary operators are given by [19]

$$
\overline{\mathcal{E}}\left(z_{1}, z_{2}\right)=\mathcal{P} \mathcal{E}_{\mathcal{A}}\left(z_{2}, z_{1}\right), \quad \overline{\mathcal{F}}\left(z_{1}, z_{2}\right)=\overline{\mathcal{E}}^{T}\left(z_{1}, z_{2}\right),
$$

where it is now understood that $z_{1}$ and $z_{2}$ correspond to the original fusion condition (2.20). These complementary operators, together with the original operators (2.25) and (2.28), satisfy the relations (2.12) and (2.13).

We have verified numerically that the complementary fused R-matrix obtained following (2.15), up to a similarity transformation, is proportional to the complex conjugate of $S^{A B}$ in [17]

$$
\left[\frac{S^{A B}\left(z_{1}, z_{23}\right)}{a_{3}\left(z_{1}, z_{2}\right) a_{3}\left(z_{1}, z_{3}\right)}\right]^{*}=\bar{R}_{1\langle\overline{23\rangle}}^{\prime}\left(z_{1} \mid z_{2}, z_{3}\right)
$$

as expected for the antisymmetric representation [32]. Again, a similar result was obtained in $[19]$.

\section{Boundary fusion}

We now generalize the above discussion to the case of boundary scattering. Let $K(z)$

$$
K(z): \quad \mathcal{C}^{n} \mapsto \mathcal{C}^{n}
$$

be a solution of the boundary Yang-Baxter equation [5-7]

$$
R_{12}\left(z_{1}, z_{2}\right) K_{1}\left(z_{1}\right) R_{21}\left(z_{2},-z_{1}\right) K_{2}\left(z_{2}\right)=K_{2}\left(z_{2}\right) R_{12}\left(z_{1},-z_{2}\right) K_{1}\left(z_{1}\right) R_{21}\left(-z_{2},-z_{1}\right),
$$


where $R_{21}\left(z_{1}, z_{2}\right)=\mathcal{P}_{12} R_{12}\left(z_{1}, z_{2}\right) \mathcal{P}_{12}, K_{1}(z)=K(z) \otimes \mathbb{I}_{n}$ and $K_{2}(z)=\mathcal{P}_{12} K_{1}(z) \mathcal{P}_{12}$.

We propose that the fused K-matrix is given by (cf. eq. (3.5) in [9])

$$
K_{\langle 12\rangle}\left(z_{1}, z_{2}\right)=\mathcal{F}_{\langle 12\rangle}\left(z_{1}, z_{2}\right) K_{1}\left(z_{1}\right) R_{21}\left(z_{2},-z_{1}\right) K_{2}\left(z_{2}\right) \mathcal{P}_{12} \mathcal{E}_{\langle 12\rangle}\left(-z_{2},-z_{1}\right) .
$$

Indeed, we show in appendix B that this object satisfies the fused boundary Yang-Baxter equation

$$
\begin{aligned}
& R_{\langle 12\rangle 3}\left(z_{1}, z_{2} \mid z_{3}\right) K_{\langle 12\rangle}\left(z_{1}, z_{2}\right) R_{3\langle 12\rangle}\left(z_{3} \mid-z_{2},-z_{1}\right) K_{3}\left(z_{3}\right) \\
& \quad=K_{3}\left(z_{3}\right) R_{\langle 12\rangle}\left(z_{1}, z_{2} \mid-z_{3}\right) K_{\langle 12\rangle}\left(z_{1}, z_{2}\right) R_{3\langle 12\rangle}\left(-z_{3} \mid-z_{2},-z_{1}\right),
\end{aligned}
$$

where $R_{\langle 12\rangle 3}$ and $R_{1\langle 23\rangle}$ are given by (2.8) and (2.9), respectively. The boundary fusion formula (3.2) is the main result of this paper. Performing a similarity transformation $\mathcal{E} \rightarrow \mathcal{E} W^{-1}, \mathcal{F} \rightarrow W \mathcal{F}$ as in (2.10) and (2.11) gives

$$
K_{\langle 12\rangle}^{\prime}\left(z_{1}, z_{2}\right)=W_{\langle 12\rangle}\left(z_{1}, z_{2}\right) K_{\langle 12\rangle}\left(z_{1}, z_{2}\right) W_{\langle 12\rangle}^{-1}\left(z_{1}, z_{2}\right) .
$$

Using the complementary operators $\overline{\mathcal{E}}$ and $\overline{\mathcal{F}}$ satisfying (2.12) and (2.13), complementary fused boundary K-matrices can be constructed in a similar manner

$$
\bar{K}_{\langle\overline{12\rangle}}\left(z_{1}, z_{2}\right)=\overline{\mathcal{F}}_{\langle\overline{12\rangle}}\left(z_{1}, z_{2}\right) K_{1}\left(z_{1}\right) R_{21}\left(z_{2},-z_{1}\right) K_{2}\left(z_{2}\right) \mathcal{P}_{12} \overline{\mathcal{E}}_{\langle\overline{12}\rangle}\left(-z_{2},-z_{1}\right) .
$$

The proof of this result is sketched in appendix B.

\subsection{AdS/CFT boundary S-matrix: symmetric representation}

Let us illustrate the boundary fusion formula (3.4) with the simplest AdS/CFT boundary S-matrix

$$
K(z)=\operatorname{diag}\left(e^{-i p / 2},-e^{i p / 2}, 1,1\right),
$$

corresponding to a $Y=0$ brane [21]. Using our previous expressions for $\mathcal{E}$ and $\mathcal{F}(2.25),(2.28)$, we obtain (cf. (2.31))

$$
K_{\langle 12\rangle}^{\prime}\left(z_{1}, z_{2}\right)=U_{\langle 12\rangle}\left(z_{1}, z_{2}\right) \tilde{\mathcal{F}}_{\langle 12\rangle}\left(z_{1}, z_{2}\right) K_{1}\left(z_{1}\right) R_{21}\left(z_{2},-z_{1}\right) K_{2}\left(z_{2}\right) \mathcal{P}_{12} \tilde{\mathcal{E}}_{\langle 12\rangle}\left(-z_{2},-z_{1}\right) T_{\langle 12\rangle}^{-1}\left(z_{1}, z_{2}\right),
$$

where $U$ is defined in (2.32), and $T$ is the following new matrix

$$
T\left(z_{1}, z_{2}\right)=W\left(z_{1}, z_{2}\right) N\left(-z_{2},-z_{1}\right)=\left.W\left(z_{1}, z_{2}\right)\right|_{w_{i} \rightarrow t_{i}},
$$

which also has the same matrix structure as $W$, but has different matrix elements. We find that these matrix elements are given by

$$
\begin{aligned}
& t_{1}=w_{1}\left(z_{1}, z_{2}\right) n_{1}\left(-z_{2},-z_{1}\right)=v_{1}, \\
& t_{2}=w_{2}\left(z_{1}, z_{2}\right) n_{2}\left(-z_{2},-z_{1}\right)=e^{-i p_{1} / 2} \frac{\eta\left(z_{1}, 1\right)}{\eta\left(z_{2}, 1\right)} v_{2},
\end{aligned}
$$

where $v_{1}$ and $v_{2}$ are given in (2.33). Using these results to evaluate (3.7), we have verified that this fused K-matrix coincides (up to an overall scalar factor) with the bound-state $Y=0$ boundary S-matrix $R^{B}$ in [24],

$$
R^{B}\left(z_{12}\right)=e^{i P / 2} K_{\langle 12\rangle}^{\prime}\left(z_{1}, z_{2}\right)
$$




$$
=\left(\begin{array}{cccccccc}
r_{1} & 0 & 0 & 0 & 0 & 0 & 0 & 0 \\
0 & r_{2} & 0 & r_{5} & 0 & 0 & 0 & 0 \\
0 & 0 & r_{3} & 0 & 0 & 0 & 0 & 0 \\
0 & r_{6} & 0 & r_{4} & 0 & 0 & 0 & 0 \\
0 & 0 & 0 & 0 & r_{7} & 0 & 0 & 0 \\
0 & 0 & 0 & 0 & 0 & r_{7} & 0 & 0 \\
0 & 0 & 0 & 0 & 0 & 0 & r_{8} & 0 \\
0 & 0 & 0 & 0 & 0 & 0 & 0 & r_{8}
\end{array}\right),
$$

where

$$
\begin{array}{lc}
r_{1}=1, \quad r_{2}=-\frac{\frac{1}{y^{-}}+y^{-}}{\frac{1}{y^{+}}+y^{-}}, & r_{3}=e^{i P}, \quad r_{4}=\frac{\frac{1}{y^{+}}+y^{+}}{\frac{1}{y^{+}}+y^{-}}, \\
r_{5}=-r_{6}=e^{i P / 2} \frac{y^{-}-y^{+}}{1+y^{-} y^{+}}, & r_{7}=-r_{8}=e^{i P / 2} .
\end{array}
$$

While the verification of some of the matrix elements is straightforward (e.g., $r_{3}$ requires just (2.24), and $r_{2}$ requires use of (2.20) and (2.21)), those involving $\eta$ 's are much more complicated. Nevertheless, by using the expression for $\eta$ in terms of a square root (A.5) and using PowerExpand in Mathematica, we managed to explicitly check all of the matrix elements.

\subsection{AdS/CFT boundary S-matrix: antisymmetric representation}

Computing the complementary fused K-matrix (3.5) using the complementary operators (2.41), as well as the fundamental bulk (2.16) and boundary (3.6) S-matrices, we obtain the diagonal matrix

$$
\bar{K}_{\langle\overline{12\rangle}\rangle}\left(z_{1}, z_{2}\right)=-\frac{\cos \left(p_{2} / 2\right)}{\cos \left(p_{1} / 2\right)} \operatorname{diag}\left(1,1,1,-1,-e^{i P / 2}, e^{-i P / 2},-e^{i P / 2}, e^{-i P / 2}\right),
$$

which satisfies the fused boundary Yang-Baxter equation (3.3) with the complementary fused R-matrices $\bar{R}_{\langle\overline{12}\rangle 3}$ and $\bar{R}_{3\langle\overline{12}\rangle}$. The result (3.12) can be related by a similarity transformation, up to an overall scalar factor, to the antisymmetric representation $M=2$ bound-state boundary S-matrix $\mathcal{R}_{2}$ obtained in [23].

\section{Discussion}

We have found a fusion formula (3.2) that is applicable to AdS/CFT boundary S-matrices, many examples of which are now known. We have focused on the $Y=0$ example only for simplicity. Although we have used the fusion formula to obtain only the $M=2$ boundstate $Y=0$ boundary S-matrices, we expect that a further generalization (along the lines of [10]) is possible for recovering the higher $(M>2)$ bound-state boundary S-matrices found in [23] and [25] for antisymmetric and symmetric representations, respectively.

We have noticed that the expressions generated by both the bulk and boundary fusion formulas are generally very complicated, and require considerable effort to simplify, particularly in the symmetric representation. It would be interesting to find a more efficient way of writing the basic elements $(\mathrm{R}, \mathcal{E}$ and $\mathcal{F})$ that lead directly to simpler results for the fused quantities. 


\section{Acknowledgments}

The work of RN was supported in part by the National Science Foundation under Grant PHY-1212337, and by a Cooper fellowship. RP thanks the Sao Paulo Research Foundation (FAPESP), grants \# 2014/00453-8 and \# 2014/20364-0, for financial support.

\section{A Fundamental bulk S-matrix}

The graded bulk S-matrix for a pair of particles in the fundamental (4-dimensional) representation of $s u(2 \mid 2)$ is given by [17]

$$
S^{A A}\left(z_{1}, z_{2}\right)=\sum_{k=1}^{10} a_{k}\left(z_{1}, z_{2}\right) \Lambda_{k}
$$

where the $16 \times 16$ matrices $\Lambda_{1}, \ldots, \Lambda_{10}$ are given in terms of quantities $E_{k i l j}$ defined by

$$
E_{k i l j}=e_{k i} \otimes e_{l j}
$$

with indices that run from 1 to 4 .

Hence, $S^{A A}\left(z_{1}, z_{2}\right)$ has the following matrix structure

$$
\left(\begin{array}{cccccccccccccccc}
a_{1} & 0 & 0 & 0 & 0 & 0 & 0 & 0 & 0 & 0 & 0 & 0 & 0 & 0 & 0 & 0 \\
0 & \frac{a_{1}}{2}+\frac{a_{2}}{2} & 0 & 0 & \frac{a_{1}}{2}-\frac{a_{2}}{2} & 0 & 0 & 0 & 0 & 0 & 0 & a_{7} & 0 & 0 & -a_{7} & 0 \\
0 & 0 & a_{5} & 0 & 0 & 0 & 0 & 0 & a_{9} & 0 & 0 & 0 & 0 & 0 & 0 & 0 \\
0 & 0 & 0 & a_{5} & 0 & 0 & 0 & 0 & 0 & 0 & 0 & 0 & a_{9} & 0 & 0 & 0 \\
0 & \frac{a_{1}}{2}-\frac{a_{2}}{2} & 0 & 0 & \frac{a_{1}}{2}+\frac{a_{2}}{2} & 0 & 0 & 0 & 0 & 0 & 0 & -a_{7} & 0 & 0 & a_{7} & 0 \\
0 & 0 & 0 & 0 & 0 & a_{1} & 0 & 0 & 0 & 0 & 0 & 0 & 0 & 0 & 0 & 0 \\
0 & 0 & 0 & 0 & 0 & 0 & a_{5} & 0 & 0 & a_{9} & 0 & 0 & 0 & 0 & 0 & 0 \\
0 & 0 & 0 & 0 & 0 & 0 & 0 & a_{5} & 0 & 0 & 0 & 0 & 0 & a_{9} & 0 & 0 \\
0 & 0 & a_{10} & 0 & 0 & 0 & 0 & 0 & a_{6} & 0 & 0 & 0 & 0 & 0 & 0 & 0 \\
0 & 0 & 0 & 0 & 0 & 0 & a_{10} & 0 & 0 & a_{6} & 0 & 0 & 0 & 0 & 0 & 0 \\
0 & 0 & 0 & 0 & 0 & 0 & 0 & 0 & 0 & 0 & a_{3} & 0 & 0 & 0 & 0 & 0 \\
0 & a_{8} & 0 & 0 & -a_{8} & 0 & 0 & 0 & 0 & 0 & 0 & \frac{a_{3}}{2}+\frac{a_{4}}{2} & 0 & 0 & \frac{a_{3}}{2}-\frac{a_{4}}{2} & 0 \\
0 & 0 & 0 & a_{10} & 0 & 0 & 0 & 0 & 0 & 0 & 0 & 0 & a_{6} & 0 & 0 & 0 \\
0 & 0 & 0 & 0 & 0 & 0 & 0 & a_{10} & 0 & 0 & 0 & 0 & 0 & a_{6} & 0 & 0 \\
0 & -a_{8} & 0 & 0 & a_{8} & 0 & 0 & 0 & 0 & 0 & 0 & \frac{a_{3}}{2}-\frac{a_{4}}{2} & 0 & 0 & \frac{a_{3}}{2}+\frac{a_{4}}{2} & 0 \\
0 & 0 & 0 & 0 & 0 & 0 & 0 & 0 & 0 & 0 & 0 & 0 & 0 & 0 & 0 & a_{3}
\end{array}\right)
$$

and the matrix elements $a_{k}=a_{k}\left(z_{1}, z_{2}\right)$ are given by [17]

$$
\begin{aligned}
& a_{1}=1, \\
& a_{2}=2 \frac{\left(x_{1}^{+}-x_{2}^{+}\right)\left(x_{1}^{-} x_{2}^{+}-1\right) x_{2}^{-}}{\left(x_{1}^{+}-x_{2}^{-}\right)\left(x_{1}^{-} x_{2}^{-}-1\right) x_{2}^{+}}-1, \\
& a_{3}=\frac{x_{2}^{+}-x_{1}^{-}}{x_{2}^{-}-x_{1}^{+}} \frac{\tilde{\eta}_{1}}{\eta_{1} \eta_{2}},
\end{aligned}
$$




$$
\begin{aligned}
a_{4} & =\frac{\left(x_{1}^{-}-x_{2}^{+}\right)}{\left(x_{2}^{-}-x_{1}^{+}\right)} \frac{\left.\tilde{\eta}_{1}\right)}{\eta_{1} \eta_{2}}-2 \frac{\left(x_{2}^{-} x_{1}^{+}-1\right)\left(x_{1}^{+}-x_{2}^{+}\right) x_{1}^{-}}{\left(x_{1}^{-} x_{2}^{-}-1\right)\left(x_{2}^{-}-x_{1}^{+}\right) x_{1}^{+}} \frac{\tilde{\eta}_{2}}{\eta_{1} \eta_{2}}, \\
a_{5} & =\frac{x_{2}^{-}-x_{1}^{-}}{x_{2}^{-}-x_{1}^{+}} \frac{\tilde{\eta}_{2}}{\eta_{2}} \\
a_{6} & =\frac{x_{1}^{+}-x_{2}^{+}}{x_{1}^{+}-x_{2}^{-}} \frac{\tilde{\eta}_{1}}{\eta_{1}} \\
a_{7} & =-\frac{i\left(x_{1}^{-}-x_{1}^{+}\right)\left(x_{2}^{-}-x_{2}^{+}\right)\left(x_{1}^{+}-x_{2}^{+}\right)}{\left(x_{1}^{-} x_{2}^{-}-1\right)\left(x_{2}^{-}-x_{1}^{+}\right)} \frac{1}{\eta_{1} \eta_{2}}, \\
a_{8} & =\frac{i x_{1}^{-} x_{2}^{-}\left(x_{1}^{+}-x_{2}^{+}\right)}{\left(x_{1}^{-} x_{2}^{-}-1\right)\left(x_{2}^{-}-x_{1}^{+}\right) x_{1}^{+} x_{2}^{+}} \tilde{\eta}_{1} \tilde{\eta}_{2}, \\
a_{9} & =\frac{x_{1}^{+}-x_{1}^{-}}{x_{1}^{+}-x_{2}^{-}} \frac{\tilde{\eta}_{2}}{\eta_{1}} \\
a_{10} & =\frac{x_{2}^{-}-x_{2}^{+}}{x_{2}^{-}-x_{1}^{+}} \frac{\tilde{\eta}_{1}}{\eta_{2}}
\end{aligned}
$$

Moreover,

$$
\eta_{1}=e^{i p_{2} / 2} \eta\left(z_{1}\right), \quad \eta_{2}=\eta\left(z_{2}\right), \quad \tilde{\eta}_{1}=\eta\left(z_{1}\right), \quad \tilde{\eta}_{2}=e^{i p_{1} / 2} \eta\left(z_{2}\right),
$$

where $\eta(z)=\eta(z, 1)$, with

$$
\eta(z, M)=e^{i p / 4} \sqrt{i\left(x^{-}-x^{+}\right)}=\sqrt{\frac{2 M}{g}} \frac{\operatorname{dn} \frac{z}{2}\left(\operatorname{cn} \frac{z}{2}+i \operatorname{sn} \frac{z}{2} \operatorname{dn} \frac{z}{2}\right)}{1+\frac{4 g^{2}}{M^{2}} \operatorname{sn}^{4} \frac{z}{2}} .
$$

\section{B Proof of the boundary fusion formulas}

We first show here that the fused K-matrix (3.2) satisfies the fused boundary Yang-Baxter equation (3.3). We use here the following shorthand notation,

$$
\begin{aligned}
& R_{12}\left(z_{1}, z_{2}\right)=R_{12}, \\
& R_{13}\left(z_{1}, z_{3}\right)=R_{13}, \\
& R_{23}\left(z_{2}, z_{3}\right)=R_{23}, \\
& \mathcal{E}_{\langle 12\rangle}\left(z_{1}, z_{2}\right)=\mathcal{E}_{\langle 12\rangle}, \\
& \mathcal{F}_{\langle 12\rangle}\left(z_{1}, z_{2}\right)=\mathcal{F}_{\langle 12\rangle}, \\
& \mathcal{H}_{\langle 12\rangle}\left(z_{1}, z_{2}\right)=\mathcal{H}_{\langle 12\rangle} \text {, }
\end{aligned}
$$

and

$$
\begin{aligned}
& R_{21}\left(z_{2},-z_{1}\right)=R_{21} \text {, } \\
& R_{31}\left(z_{3},-z_{1}\right)=R_{31}, \\
& R_{32}\left(z_{3},-z_{2}\right)=R_{32} \text {, } \\
& K_{1}\left(z_{1}\right)=K_{1} \text {, } \\
& K_{2}\left(z_{2}\right)=K_{2}, \\
& K_{3}\left(z_{3}\right)=K_{3} .
\end{aligned}
$$

If the arguments of a given matrix do not fit the above notation we write them explicitly.

$$
\begin{aligned}
& \mathcal{H}_{\langle 12\rangle}\left(z_{1}, z_{2}\right) R_{\langle 12\rangle 3}\left(z_{1}, z_{2} \mid z_{3}\right) K_{\langle 12\rangle}\left(z_{1}, z_{2}\right) R_{3\langle 12\rangle}\left(z_{3} \mid-z_{2},-z_{1}\right) K_{3}\left(z_{3}\right) \\
& =\underbrace{\mathcal{H}_{\langle 12\rangle} \mathcal{F}_{\langle 12\rangle}}_{\text {Eq. }(2.6)} R_{13} R_{23} \mathcal{E}_{\langle 12\rangle} \mathcal{F}_{\langle 12\rangle} K_{1} R_{21} K_{2} \mathcal{P}_{12} \mathcal{E}_{\langle 12\rangle}\left(-z_{2},-z_{1}\right) \mathcal{F}_{\langle 12\rangle}\left(-z_{2},-z_{1}\right) R_{32}\left(z_{3},-z_{1}\right) \cdots \\
& \quad \cdots R_{31}\left(z_{3},-z_{2}\right) \underbrace{\mathcal{E}_{\langle 12\rangle}\left(-z_{2},-z_{1}\right) K_{3}}_{\text {commute }}
\end{aligned}
$$




$$
\begin{aligned}
& =\mathcal{F}_{\langle 12\rangle} \underbrace{R_{12} R_{13} R_{23}}_{\text {Eq. }(2.1)} \mathcal{E}_{\langle 12\rangle} \mathcal{F}_{\langle 12\rangle} K_{1} R_{21} K_{2} \mathcal{P}_{12} \mathcal{E}_{\langle 12\rangle}\left(-z_{2},-z_{1}\right) \mathcal{F}_{\langle 12\rangle}\left(-z_{2},-z_{1}\right) R_{32}\left(z_{3},-z_{1}\right) \cdots \\
& \cdots R_{31}\left(z_{3},-z_{2}\right) K_{3} \mathcal{E}_{\langle 12\rangle}\left(-z_{2},-z_{1}\right) \\
& =\mathcal{F}_{\langle 12\rangle} R_{23} R_{13} \underbrace{R_{12} \mathcal{E}_{\langle 12\rangle} \mathcal{F}_{\langle 12\rangle}}_{\text {Eq. }(2.7)} K_{1} R_{21} K_{2} \mathcal{P}_{12} \mathcal{E}_{\langle 12\rangle}\left(-z_{2},-z_{1}\right) \mathcal{F}_{\langle 12\rangle}\left(-z_{2},-z_{1}\right) R_{32}\left(z_{3},-z_{1}\right) \cdots \\
& \cdots R_{31}\left(z_{3},-z_{2}\right) K_{3} \mathcal{E}_{\langle 12\rangle}\left(-z_{2},-z_{1}\right) \\
& =\mathcal{F}_{\langle 12\rangle} R_{23} R_{13} \underbrace{R_{12} K_{1} R_{21} K_{2}}_{\text {Eq. }(3.1)} \mathcal{P}_{12} \mathcal{E}_{\langle 12\rangle}\left(-z_{2},-z_{1}\right) \mathcal{F}_{\langle 12\rangle}\left(-z_{2},-z_{1}\right) R_{32}\left(z_{3},-z_{1}\right) \cdots \\
& \cdots R_{31}\left(z_{3},-z_{2}\right) K_{3} \mathcal{E}_{\langle 12\rangle}\left(-z_{2},-z_{1}\right) \\
& =\mathcal{F}_{\langle 12\rangle} R_{23} \underbrace{R_{13} K_{2}}_{\text {commute }} R_{12}\left(z_{1},-z_{2}\right) K_{1} \underbrace{R_{21}\left(-z_{2},-z_{1}\right) \mathcal{P}_{12}}_{\mathcal{P}_{12}^{2}=1} \mathcal{E}_{\langle 12\rangle}\left(-z_{2},-z_{1}\right) \mathcal{F}_{\langle 12\rangle}\left(-z_{2},-z_{1}\right) \cdots \\
& \cdots R_{32}\left(z_{3},-z_{1}\right) R_{31}\left(z_{3},-z_{2}\right) K_{3} \mathcal{E}_{\langle 12\rangle}\left(-z_{2},-z_{1}\right) \\
& =\mathcal{F}_{\langle 12\rangle} R_{23} K_{2} R_{13} R_{12}\left(z_{1},-z_{2}\right) K_{1} \mathcal{P}_{12} \underbrace{R_{12}\left(-z_{2},-z_{1}\right) \mathcal{E}_{\langle 12\rangle}\left(-z_{2},-z_{1}\right) \mathcal{F}_{\langle 12\rangle}\left(-z_{2},-z_{1}\right)}_{\text {Eq. }(2.7)} \cdots \\
& \cdots R_{32}\left(z_{3},-z_{1}\right) R_{31}\left(z_{3},-z_{2}\right) K_{3} \mathcal{E}_{\langle 12\rangle}\left(-z_{2},-z_{1}\right) \\
& =\mathcal{F}_{\langle 12\rangle} R_{23} K_{2} R_{13} R_{12}\left(z_{1},-z_{2}\right) K_{1} \mathcal{P}_{12} \underbrace{R_{12}\left(-z_{2},-z_{1}\right) R_{32}\left(z_{3},-z_{1}\right) R_{31}\left(z_{3},-z_{2}\right)}_{\text {Eq. }(2.1)} K_{3} \cdots \\
& \cdots \mathcal{E}_{\langle 12\rangle}\left(-z_{2},-z_{1}\right) \\
& =\mathcal{F}_{\langle 12\rangle} R_{23} K_{2} R_{13} R_{12}\left(z_{1},-z_{2}\right) K_{1} \underbrace{\mathcal{P}_{12} R_{31}\left(z_{3},-z_{2}\right) R_{32}\left(z_{3},-z_{1}\right) R_{12}\left(-z_{2},-z_{1}\right)}_{\mathcal{P}_{12}^{2}=1} K_{3} \cdots \\
& \cdots \mathcal{E}_{\langle 12\rangle}\left(-z_{2},-z_{1}\right) \\
& =\mathcal{F}_{\langle 12\rangle} R_{23} K_{2} R_{13} R_{12}\left(z_{1},-z_{2}\right) \underbrace{K_{1} R_{32}}_{\text {commute }} R_{31} \underbrace{R_{21}\left(-z_{2},-z_{1}\right) \mathcal{P}_{12} K_{3}}_{\text {commute }} \mathcal{E}_{\langle 12\rangle}\left(-z_{2},-z_{1}\right) \\
& =\mathcal{F}_{\langle 12\rangle} R_{23} K_{2} \underbrace{R_{13} R_{12}\left(z_{1},-z_{2}\right) R_{32}}_{\text {Eq. }(2.1)} K_{1} R_{31} K_{3} R_{21}\left(-z_{2},-z_{1}\right) \mathcal{P}_{12} \mathcal{E}_{\langle 12\rangle}\left(-z_{2},-z_{1}\right) \\
& =\mathcal{F}_{\langle 12\rangle} R_{23} K_{2} R_{32} R_{12}\left(z_{1},-z_{2}\right) \underbrace{R_{13} K_{1} R_{31} K_{3}}_{\text {Eq. }(3.1)} R_{21}\left(-z_{2},-z_{1}\right) \mathcal{P}_{12} \mathcal{E}_{\langle 12\rangle}\left(-z_{2},-z_{1}\right) \\
& =\mathcal{F}_{\langle 12\rangle} R_{23} K_{2} R_{32} \underbrace{R_{12}\left(z_{1},-z_{2}\right) K_{3}}_{\text {commute }} R_{13}\left(z_{1},-z_{3}\right) K_{1} R_{31}\left(-z_{3},-z_{1}\right) R_{21}\left(-z_{2},-z_{1}\right) \cdots \\
& \cdots \mathcal{P}_{12} \mathcal{E}_{\langle 12\rangle}\left(-z_{2},-z_{1}\right) \\
& =\mathcal{F}_{\langle 12\rangle} \underbrace{R_{23} K_{2} R_{32} K_{3}}_{\text {Eq. }(3.1)} R_{12}\left(z_{1},-z_{2}\right) R_{13}\left(z_{1},-z_{3}\right) K_{1} R_{31}\left(-z_{3},-z_{1}\right) R_{21}\left(-z_{2},-z_{1}\right) \cdots \\
& \cdots \mathcal{P}_{12} \mathcal{E}_{\langle 12\rangle}\left(-z_{2},-z_{1}\right) \\
& =\underbrace{\mathcal{F}_{\langle 12\rangle} K_{3}}_{\text {commute }} R_{23}\left(z_{2},-z_{3}\right) K_{2} \underbrace{R_{32}\left(-z_{3},-z_{2}\right) R_{12}\left(z_{1},-z_{2}\right) R_{13}\left(z_{1},-z_{3}\right)}_{\text {Eq. }(2.1)} K_{1} R_{31}\left(-z_{3},-z_{1}\right) \cdots \\
& \cdots R_{21}\left(-z_{2},-z_{1}\right) \mathcal{P}_{12} \mathcal{E}_{\langle 12\rangle}\left(-z_{2},-z_{1}\right) \\
& =K_{3} \mathcal{F}_{\langle 12\rangle} R_{23}\left(z_{2},-z_{3}\right) \underbrace{K_{2} R_{13}\left(z_{1},-z_{3}\right)}_{\text {commute }} R_{12}\left(z_{1},-z_{2}\right) \underbrace{R_{32}\left(-z_{3},-z_{2}\right) K_{1}}_{\text {commute }} R_{31}\left(-z_{3},-z_{1}\right) \cdots
\end{aligned}
$$




$$
\begin{aligned}
& \cdots R_{21}\left(-z_{2},-z_{1}\right) \mathcal{P}_{12} \mathcal{E}_{\langle 12\rangle}\left(-z_{2},-z_{1}\right) \\
& =K_{3} \mathcal{F}_{\langle 12\rangle} R_{23}\left(z_{2},-z_{3}\right) R_{13}\left(z_{1},-z_{3}\right) K_{2} R_{12}\left(z_{1},-z_{2}\right) K_{1} \cdots \\
& \cdots \underbrace{R_{32}\left(-z_{3},-z_{2}\right) R_{31}\left(-z_{3},-z_{1}\right) R_{21}\left(-z_{2},-z_{1}\right)}_{\text {Eq. }(2.1)} \mathcal{P}_{12} \mathcal{E}_{\langle 12\rangle}\left(-z_{2},-z_{1}\right) \\
& =K_{3} \mathcal{F}_{\langle 12\rangle} R_{23}\left(z_{2},-z_{3}\right) R_{13}\left(z_{1},-z_{3}\right) K_{2} R_{12}\left(z_{1},-z_{2}\right) K_{1} \cdots \\
& \cdots \underbrace{R_{21}\left(-z_{2},-z_{1}\right) R_{31}\left(-z_{3},-z_{1}\right) R_{32}\left(-z_{3},-z_{2}\right) \mathcal{P}_{12}}_{\mathcal{P}_{12}^{2}=1} \mathcal{E}_{\langle 12\rangle}\left(-z_{2},-z_{1}\right) \\
& =K_{3} \mathcal{F}_{\langle 12\rangle} R_{23}\left(z_{2},-z_{3}\right) R_{13}\left(z_{1},-z_{3}\right) K_{2} R_{12}\left(z_{1},-z_{2}\right) K_{1} \cdots \\
& \cdots \mathcal{P}_{12} \underbrace{R_{12}\left(-z_{2},-z_{1}\right)}_{\text {Eq. }(2.7) \text { and } \mathcal{P}_{12}^{2}=1} R_{32}\left(-z_{3},-z_{1}\right) R_{31}\left(-z_{3},-z_{2}\right) \mathcal{E}_{\langle 12\rangle}\left(-z_{2},-z_{1}\right) \\
& =K_{3} \mathcal{F}_{\langle 12\rangle} R_{23}\left(z_{2},-z_{3}\right) R_{13}\left(z_{1},-z_{3}\right) \underbrace{K_{2} R_{12}\left(z_{1},-z_{2}\right) K_{1} R_{21}\left(-z_{2},-z_{1}\right)}_{\text {Eq. }(3.1)} \mathcal{P}_{12} \mathcal{E}_{\langle 12\rangle}\left(-z_{2},-z_{1}\right) \cdots \\
& \cdots \mathcal{F}_{\langle 12\rangle}\left(-z_{2},-z_{1}\right) R_{32}\left(-z_{3},-z_{1}\right) R_{31}\left(-z_{3},-z_{2}\right) \mathcal{E}_{\langle 12\rangle}\left(-z_{2},-z_{1}\right) \\
& =K_{3} \mathcal{F}_{\langle 12\rangle} R_{23}\left(z_{2},-z_{3}\right) R_{13}\left(z_{1},-z_{3}\right) \underbrace{R_{12}} K_{1} R_{21} K_{2} \mathcal{P}_{12} \mathcal{E}_{\langle 12\rangle}\left(-z_{2},-z_{1}\right) R_{3\langle 12\rangle}\left(-z_{3} \mid-z_{2},-z_{1}\right) \\
& \text { Eq.(2.7) } \\
& =K_{3} \mathcal{F}_{\langle 12\rangle} \underbrace{R_{23}\left(z_{2},-z_{3}\right) R_{13}\left(z_{1},-z_{3}\right) R_{12}}_{\text {Eq. }(2.1)} \mathcal{E}_{\langle 12\rangle} K_{\langle 12\rangle}\left(z_{1}, z_{2}\right) R_{3\langle 12\rangle}\left(-z_{3} \mid-z_{2},-z_{1}\right) \\
& =K_{3} \underbrace{\mathcal{F}_{\langle 12\rangle} R_{12}}_{\text {Eq. }(2.6)} R_{13}\left(z_{1},-z_{3}\right) R_{23}\left(z_{2},-z_{3}\right) \mathcal{E}_{\langle 12\rangle} K_{\langle 12\rangle}\left(z_{1}, z_{2}\right) R_{3\langle 12\rangle}\left(-z_{3} \mid-z_{2},-z_{1}\right) \\
& =\underbrace{K_{3} \mathcal{H}_{\langle 12\rangle}}_{\text {commute }} \mathcal{F}_{\langle 12\rangle} R_{13}\left(z_{1},-z_{3}\right) R_{23}\left(z_{2},-z_{3}\right) \mathcal{E}_{\langle 12\rangle} K_{\langle 12\rangle}\left(z_{1}, z_{2}\right) R_{3\langle 12\rangle}\left(-z_{3} \mid-z_{2},-z_{1}\right) \\
& =\mathcal{H}_{\langle 12\rangle}\left(z_{1}, z_{2}\right) K_{3}\left(z_{3}\right) R_{\langle 12\rangle 3}\left(z_{1}, z_{2} \mid-z_{3}\right) K_{\langle 12\rangle}\left(z_{1}, z_{2}\right) R_{3\langle 12\rangle}\left(-z_{3} \mid-z_{2},-z_{1}\right)
\end{aligned}
$$

The proof of the complementary boundary fusion formula (3.5) is similar to the one for the bulk [19]. In particular, one first needs the identity

$$
\mathcal{F}_{\langle 12\rangle} K_{1} R_{21}\left(z_{2},-z_{1}\right) K_{2} \mathcal{P}_{12} \overline{\mathcal{E}}_{\langle\overline{12}\rangle}\left(-z_{2},-z_{1}\right)=0,
$$

whose proof is as follows:

$$
\begin{aligned}
& \underbrace{\mathcal{H}_{\langle 12\rangle} \mathcal{F}_{\langle 12\rangle}}_{\text {Eq. }(2.6)} K_{1} R_{21} K_{2} \mathcal{P}_{12} \overline{\mathcal{E}}_{\langle\overline{12}\rangle}\left(-z_{2},-z_{1}\right) \\
& =\mathcal{F}_{\langle 12\rangle} \underbrace{R_{12} K_{1} R_{21} K_{2}}_{\text {Eq. }(3.1)} \mathcal{P}_{12} \overline{\mathcal{E}}_{\langle\overline{12}\rangle}\left(-z_{2},-z_{1}\right) \\
& =\mathcal{F}_{\langle 12\rangle} K_{2} R_{12}\left(z_{1},-z_{2}\right) K_{1} \underbrace{R_{21}\left(-z_{2},-z_{1}\right) \mathcal{P}_{12}}_{\mathcal{P}_{12}^{2}=1} \overline{\mathcal{E}}_{\langle\overline{12\rangle}\rangle}\left(-z_{2},-z_{1}\right) \\
& =\mathcal{F}_{\langle 12\rangle} K_{2} R_{12}\left(z_{1},-z_{2}\right) K_{1} \mathcal{P}_{12} R_{12}\left(-z_{2},-z_{1}\right) \overline{\mathcal{E}}_{\langle\overline{12}\rangle}\left(-z_{2},-z_{1}\right)=0 .
\end{aligned}
$$

In passing to the final equality, we used the fact $R\left(z_{1}, z_{2}\right) \overline{\mathcal{E}}\left(z_{1}, z_{2}\right)=0$, which is a direct consequence of the decomposition (2.3) and the orthogonality relation $\mathcal{F}\left(z_{1}, z_{2}\right) \overline{\mathcal{E}}\left(z_{1}, z_{2}\right)=0(2.12)$. 
It follows from (B.2) and the completeness relation (2.13) that

$$
\begin{aligned}
& \overline{\mathcal{E}}_{\langle\overline{12}\rangle} \overline{\mathcal{F}}_{\langle\overline{12}\rangle} K_{1} R_{21}\left(z_{2},-z_{1}\right) K_{2} \mathcal{P}_{12} \overline{\mathcal{E}}_{\langle\overline{12}\rangle}\left(-z_{2},-z_{1}\right) \\
& \quad=\left(1-\mathcal{E}_{\langle 12\rangle} \mathcal{F}_{\langle 12\rangle}\right) K_{1} R_{21}\left(z_{2},-z_{1}\right) K_{2} \mathcal{P}_{12} \overline{\mathcal{E}}_{\langle\overline{12}\rangle}\left(-z_{2},-z_{1}\right) \\
& \quad=K_{1} R_{21}\left(z_{2},-z_{1}\right) K_{2} \mathcal{P}_{12} \overline{\mathcal{E}}_{\langle\overline{12}\rangle}\left(-z_{2},-z_{1}\right) .
\end{aligned}
$$

In other words, the projector $\overline{\mathcal{E}}_{\langle\overline{12}\rangle} \overline{\mathcal{F}}_{\langle\overline{12}\rangle}$ can be inserted or removed in front of

$$
K_{1} R_{21}\left(z_{2},-z_{1}\right) K_{2} \mathcal{P}_{12} \overline{\mathcal{E}}_{\langle\overline{12}\rangle}\left(-z_{2},-z_{1}\right)
$$

as needed. Armed with this fact, together with the corresponding bulk result [19]

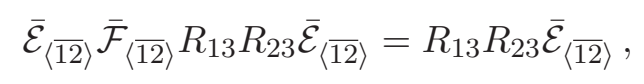

it is now a somewhat long but straightforward calculation to verify that the complementary fused boundary K-matrix (3.5) obeys the fused boundary Yang-Baxter equation

$$
\begin{aligned}
& \bar{R}_{\langle\overline{12}\rangle 3}\left(z_{1}, z_{2} \mid z_{3}\right) \bar{K}_{\langle\overline{12}\rangle}\left(z_{1}, z_{2}\right) \bar{R}_{3\langle\overline{12}\rangle}\left(z_{3} \mid-z_{2},-z_{1}\right) K_{3}\left(z_{3}\right) \\
& \quad=K_{3}\left(z_{3}\right) \bar{R}_{\langle\overline{12}\rangle 3}\left(z_{1}, z_{2} \mid-z_{3}\right) \bar{K}_{\langle\overline{12}\rangle}\left(z_{1}, z_{2}\right) \bar{R}_{3\langle\overline{12}\rangle}\left(-z_{3} \mid-z_{2},-z_{1}\right),
\end{aligned}
$$

where the complementary fused R-matrices are given by (2.14) and (2.15).

Open Access. This article is distributed under the terms of the Creative Commons Attribution License (CC-BY 4.0), which permits any use, distribution and reproduction in any medium, provided the original author(s) and source are credited.

\section{References}

[1] A.B. Zamolodchikov and A.B. Zamolodchikov, Factorized $S$ matrices in two-dimensions as the exact solutions of certain relativistic quantum field models, Annals Phys. 120 (1979) 253 [INSPIRE].

[2] M. Karowski, On the bound state problem in $(1+1)$-dimensional field theories, Nucl. Phys. B 153 (1979) 244 [INSPIRE].

[3] P.P. Kulish, N. Yu. Reshetikhin and E.K. Sklyanin, Yang-Baxter equation and representation theory. 1, Lett. Math. Phys. 5 (1981) 393 [InSPIRE].

[4] P.P. Kulish and E.K. Sklyanin, Quantum spectral transform method. Recent developments, Lect. Notes Phys. 151 (1982) 61 [INSPIRE].

[5] I.V. Cherednik, Factorizing particles on a half line and root systems, Theor. Math. Phys. 61 (1984) 977 [InSPIRE].

[6] E.K. Sklyanin, Boundary conditions for integrable quantum systems, J. Phys. A 21 (1988) 2375 [inSPIRE].

[7] S. Ghoshal and A.B. Zamolodchikov, Boundary $S$ matrix and boundary state in two-dimensional integrable quantum field theory, Int. J. Mod. Phys. A 9 (1994) 3841 [Erratum ibid. A 9 (1994) 4353] [hep-th/9306002] [INSPIRE]. 
[8] L. Mezincescu, R.I. Nepomechie and V. Rittenberg, Bethe ansatz solution of the Fateev-Zamolodchikov quantum spin chain with boundary terms, Phys. Lett. A 147 (1990) 70 [INSPIRE].

[9] L. Mezincescu and R.I. Nepomechie, Fusion procedure for open chains, J. Phys. A 25 (1992) 2533 [inSPIRE].

[10] Y.-K. Zhou, Row transfer matrix functional relations for Baxter's eight vertex and six vertex models with open boundaries via more general reflection matrices, Nucl. Phys. B 458 (1996) 504 [hep-th/9510095] [InSPIRE].

[11] A. Kuniba, T. Nakanishi and J. Suzuki, T-systems and Y-systems in integrable systems, J. Phys. A 44 (2011) 103001 [arXiv:1010.1344] [inSPIRE].

[12] M. Staudacher, The factorized S-matrix of CFT/AdS, JHEP 05 (2005) 054 [hep-th/0412188] [INSPIRE].

[13] N. Beisert, The SU(2|2) dynamic S-matrix, Adv. Theor. Math. Phys. 12 (2008) 945 [hep-th/0511082] [INSPIRE].

[14] N. Beisert et al., Review of AdS/CFT integrability: an overview, Lett. Math. Phys. 99 (2012) 3 [arXiv:1012.3982] [INSPIRE].

[15] N. Dorey, Magnon bound states and the AdS/CFT correspondence, J. Phys. A 39 (2006) 13119 [hep-th/0604175] [InSPIRE].

[16] G. Arutyunov and S. Frolov, On string S-matrix, bound states and TBA, JHEP 12 (2007) 024 [arXiv:0710.1568] [INSPIRE].

[17] G. Arutyunov and S. Frolov, The S-matrix of string bound states, Nucl. Phys. B 804 (2008) 90 [arXiv:0803.4323] [inSPIRE].

[18] G. Arutyunov, M. de Leeuw and A. Torrielli, The bound state $S$-matrix for $A d S_{5} \times S^{5}$ superstring, Nucl. Phys. B 819 (2009) 319 [arXiv:0902.0183] [INSPIRE].

[19] N. Beisert, M. de Leeuw and P. Nag, Fusion for the one-dimensional Hubbard model, J. Phys. A 48 (2015) 324002 [arXiv:1503.04838] [InSPIRE].

[20] F. Gohmann, A. Seel and J. Suzuki, Correlation functions of the integrable isotropic spin-1 chain at finite temperature, J. Stat. Mech. 11 (2010) P11011 [arXiv:1008.4440] [InSPIRE].

[21] D.M. Hofman and J.M. Maldacena, Reflecting magnons, JHEP 11 (2007) 063 [arXiv:0708.2272] [INSPIRE].

[22] K. Zoubos, Review of AdS/CFT integrability, chapter IV.2: deformations, orbifolds and open boundaries, Lett. Math. Phys. 99 (2012) 375 [arXiv:1012.3998] [INSPIRE].

[23] D.H. Correa and C.A.S. Young, Finite size corrections for open strings/open chains in planar AdS/CFT, JHEP 08 (2009) 097 [arXiv:0905.1700] [INSPIRE].

[24] C. Ahn and R.I. Nepomechie, Yangian symmetry and bound states in AdS/CFT boundary scattering, JHEP 05 (2010) 016 [arXiv:1003.3361] [INSPIRE].

[25] L. Palla, Yangian symmetry of boundary scattering in AdS/CFT and the explicit form of bound state reflection matrices, JHEP 03 (2011) 110 [arXiv:1102.0122] [INSPIRE].

[26] N. MacKay and V. Regelskis, Yangian symmetry of the $Y=0$ maximal giant graviton, JHEP 12 (2010) 076 [arXiv:1010.3761] [INSPIRE]. 
[27] N. MacKay and V. Regelskis, Reflection algebra, Yangian symmetry and bound-states in AdS/CFT, JHEP 01 (2012) 134 [arXiv:1101.6062] [INSPIRE].

[28] D.H. Correa, V. Regelskis and C.A.S. Young, Integrable achiral D5-brane reflections and asymptotic Bethe equations, J. Phys. A 44 (2011) 325403 [arXiv: 1105.3707] [INSPIRE].

[29] N. MacKay and V. Regelskis, Achiral boundaries and the twisted Yangian of the D5-brane, JHEP 08 (2011) 019 [arXiv: 1105.4128] [INSPIRE].

[30] V. Regelskis, The secret symmetries of the AdS/CFT reflection matrices, JHEP 08 (2011) 006 [arXiv:1105.4497] [INSPIRE].

[31] A. De La Rosa Gomez and N.J. MacKay, Twisted Yangian symmetry of the open Hubbard model, J. Phys. A 47 (2014) 305203 [arXiv: 1404. 2095] [InSPIRE].

[32] Z. Bajnok and R.A. Janik, Four-loop perturbative Konishi from strings and finite size effects for multiparticle states, Nucl. Phys. B 807 (2009) 625 [arXiv:0807.0399] [INSPIRE]. 